\title{
Ultrastructural study reveals that mouse hippocampal neurons are more protected than the cerebrocortical neurons from age related cytological alterations
}

\begin{abstract}
Neurons are post mitotic cells. They are more vulnerable to oxidative damage because of high content of unsaturated fatty acids and high oxygen consumption per unit weight. As the age of an organism progresses, the oxidative damage to molecules also increases which leads to increase in oxidatively modified molecules in the cell. Damage to membrane lipids causes alterations in the membrane functions. In the present study we have assessed the oxidative damage to membrane lipids in the form of nM of malondialdehyde (MDA) in Cerebral cortex and hippocampus of 12, 18 and 24 months old male albino mice. We found that the degree of oxidative damage was higher in Cerebral cortex than the hippocampus. This indicates that hippocampus is more resistant to oxidative damage than Cerebral cortex. We have also studied the ultrastructure of cerebrocortical and hippocampal neurons. We found that the RER, Golgi apparatus and mitochondria were well organized in hippocampal neurons, whereas in cerebrocortical neurons, these cell organelles exhibited degenerative changes at 24 months of age. Nucleus of cerebrocortical neurons exhibited predominantly heterochromatinised form, whereas in hippocampal neurons it was in Euchromatin form.
\end{abstract}

Keywords: malondialdehyde (mda), oxidative damage, lipid peroxidation, ultrastructural neuropathology
Volume 2 Issue 5 - 2015

\author{
Pathan K H,Gajare K A, Deshmukh AA \\ 'Department of Zoology, Shivaji University, Kolhapur (MS), India \\ 2Department of Zoology, The New College, Kolhapur (MS), India \\ Correspondence: Deshmukh AA, Department of Zoology, \\ Shivaji University, Kolhapur (MS), India, Tel +9123 | 2609249, \\ +919822230905, Fax 02312691533. \\ Email ashish_cellbio@rediffmail.com
}

Received: October 21, 2015 | Published: November 05, 2015
Abbreviations: ROS, reactive oxygen species; LP, lipid peroxidation; PUFAS, polyunsaturated fatty acids; MDA, malondialdehyde; HHE, 4-hydroxy- 2-hexenal; HNE, 4-hydroxy-2nonenal; $\mathrm{AD}$, alzheimer's disease; TBA, thiobarbituric acid

\section{Introduction}

The "free radical theory of aging" proposed by Harman postulates that free radicals cause damage to cellular macromolecules and thereby deteriorates cellular functions. ${ }^{1}$ The brain is more susceptible to oxidative stress due to high content of unsaturated fatty acids; high oxygen consumption per unit weight; High content of lipid peroxidation key ingredients (iron and ascorbate); and the scarcity of antioxidant defenses systems. ${ }^{2}$ Oxidative damage to the brain leads to dementia. Even smaller impairment in the neural tissue increases the susceptibility to neurodegenerative diseases such as Alzheimer's disease, Parkinson's disease, and amyotrophic lateral sclerosis. ${ }^{3}$ Neurodegenerative diseases are characterized by decline in the normal antioxidant defense mechanisms that leads to deleterious oxidative changes. ${ }^{4}$ Since, mitochondria are involved in redox reactions associated with cellular respiration, they are the source of Reactive Oxygen Species [ROS]. ${ }^{5-6}$ Superoxide radicals are generated during mitochondrial metabolism get dismutated to hydrogen peroxide ${ }^{7}$ which reacts with ferrous ions to generate most dangerous hydroxyl radicals. ${ }^{8}$ The free radicals cause oxidative damage to mitochondrial DNA and affect mitochondrial metabolism. ${ }^{9,10}$ The damaged mitochondria produce less ATP and more free radicals thereby increases the oxidative stress. ${ }^{11}$ The free radicals react with membrane lipids and cause oxidative damage. ${ }^{12,13}$ Except ribosome's, all the cell organelles are membrane bound. Thus, membranes of virtually all cell organelles are potentially targets of oxidative damage. Damage to membrane structure affects ion transporters, signal transduction, membrane potential and membrane permeability which ultimately perturb cellular metabolism that eventually leading to cell death. ${ }^{14}$ Oxidative damage to proteins cause structural modifications such as protein cross linking, change in three dimensional structure of proteins. ${ }^{15,16}$ This leads to loss of functions of the proteins. Oxidatively modified biomolecules become resistant to lysosomal degradation and get accumulated in the lysosomes as intracellular debris called as lipofuscin granules. ${ }^{17,18}$ ROS also cause oxidative damage to DNA resulting into genome instability and affect transcription. ${ }^{19,20}$ ROS and mitochondria are involved in pathogenesis of neurodegenerative diseases. ${ }^{21}$ Lipid peroxidation (LP) is the oxidative modification in the polyunsaturated fatty acids (PUFAS) ${ }^{22}$ During this process, large numbers of toxic byproducts are generated which alter the cellular functions and ultimately affect the cell survival. ${ }^{23}$

The byproducts of lipid peroxidation are chemically reactive aldehydes. They possess the neurotoxic activity e.g. Malondialdehyde (MDA), acrolein, 4-hydroxy- 2-hexenal (HHE), and 4-hydroxy2-nonenal (HNE). ${ }^{24,25}$ The free radical-mediated MDA production causes damage to the proteins as well as DNA in the brain. ${ }^{26}$ ROS contributes to Alzheimer's disease (AD). The persons with AD has shown increased levels of thiobarbiturate reactive substances in deceased brain regions as compared to age matched control. ${ }^{27}$ Age associated increase in oxidative stress, factors like accumulation of amyloid in Alzheimer's disease and iron deposition in Parkinson's disease enhance the lipid peroxidation process and generation of 
4-hydroxy-2-nonenal (HNE). ${ }^{28,29}$ The lipid peroxidation studies are more important in $\mathrm{AD}^{30,31}$ In the present investigation, we have studied the age related alterations in the MDA and Ultrastructural changes in cerebrocortical and hippocampal neurons at 12, 18 and 24months.

\section{Materials and method}

In the present investigations, male Swiss albino mice Mus musculus were used. The experiments were carried out with due permission of Institutional animal ethics committee in accordance with CPCSEA guidelines. The mice were provided with drinking water and laboratory animal feed (Pranav Agro industries, Sangli) ad libitum and maintained in animal house six animals per cage and $12 \mathrm{hrs}$ light and $12 \mathrm{hrs}$ dark cycle. The animals were sacrificed after 12 , 18 and 24 months of age. Six animals were sacrificed after stipulated age. The brain was dissected on prechilled platform, cerebral cortex and hippocampus were separated and, used for further studies.

\section{Measurement of lipid peroxidation}

Cerebral cortex and hippocampus of six animals were used after attaining the age of 12,18 and 24 months. Malondialdehyde (MDA) is the most common end product of lipid peroxidation, ${ }^{32,33}$ which was estimated spectrophotometrically by thiobarbituric acid reaction. ${ }^{34}$ In this reaction one molecule of malondialdehyde reacts with two molecules of Thiobarbituric acid (TBA) at boiling temperature to produce red colored MDA-TBA complex which was read spectrophotometrically at $532 \mathrm{~nm}$. The cerebral cortex and hippocampus were homogenized in reaction mixture containing potassium phosphate buffer $\mathrm{pH} 7.0(75 \mathrm{mM})$, ascorbic acid $(1 \mathrm{mM})$ and ferric chloride $(1 \mathrm{mM})$ at the concentration of $2 \mathrm{mg} / \mathrm{ml} .0 .2 \mathrm{ml}$ of the homogenate was mixed with $1 \mathrm{ml}$ of $20 \% \mathrm{TCA}$ and $2 \mathrm{ml}$ of $0.67 \% \mathrm{TBA}$ and kept in boiling water bath for 10min. After cooling, the optical densities were read at $532 \mathrm{~nm}$.

\section{Statistical analysis}

The results were processed as arithmetic mean \pm standard deviation of sx animals and level of significance was analyzed by 'Student $t$ test'. The levels of MDA in the hippocampus were compared with the levels of MDA in the cerebral cortex at all the ages.

\section{Transmission electron microscopic studies}

Transmission Electron Microscopy was carried out at the department of Electron Microscopy, Histopathology Unit, Jaslok hospital and research Centre, Mumbai. Approximately $1 \mathrm{~mm} \mathrm{X} 1 \mathrm{~mm}$ pieces of frontal cortex and dentate gyrus region of hippocampus were fixed in $3 \%$ glutaraldehyde for $24 \mathrm{hrs}$ at $4^{\circ} \mathrm{C}$, and washed twice with $0.1 \mathrm{M}$ cacodylate buffer $\mathrm{pH} 7.4$, each wash was for $30 \mathrm{~min}$. The pieces were transferred to $1 \%$ Osmium tetroxide for $2 \mathrm{hrs}$, washed with $0.1 \mathrm{M}$ cacodylate buffer $\mathrm{pH} 7.4$ for $20 \mathrm{~min}$. then dehydrated through $50 \%, 70 \%, 90 \%$ ethanol, for $30 \mathrm{~min}$ in each grade $(15 \mathrm{~min}+15 \mathrm{~min}$ two changes of each grade) and finally in absolute ethanol for one hour (two changes of $30 \mathrm{~min}$ ). The dehydrated pieces were transferred to propylene oxide for $30 \mathrm{~min}$ and passed through 1:1 propylene oxide and araldite (two changes of $15 \mathrm{~min}$ ), 1:3 propylene oxide and araldite (two changes of $15 \mathrm{~min}$ ) and finally embedded in araldite in Beem capsules and kept for drying at $80^{\circ} \mathrm{C}$ for $12 \mathrm{hrs}$.

After trimming the araldite blocks, semi-thin sections of 1 micron thickness were cut on Leica-UC7 Ultra microtome followed by ultrathin sectioning of $80 \mathrm{~nm}$. Each ultrathin section was collected on 300mesh copper grid (Tabb Laboratories Equipment Ltd England) and stained with $12.5 \%$ uranyl acetate for $40 \mathrm{~min}$ followed by $0.25 \%$ lead citrate for $5 \mathrm{~min}$. After drying, the sections were observed using Jeol-100S transmission electron microscope at $80 \mathrm{KV}$ voltage.

\section{Results and discussion}

\section{Levels of MDA in $\mathrm{nM} / \mathrm{mg}$ tissue in various regions of mouse brain expressed as Arithmetic mean \pm S.D}

In cerebral cortex of 12 months old mice $0.22 \pm 0.025 \mathrm{nM}$ of MDA/ mg tissue was observed. While the hippocampal MDA level was $0.083 \pm 0.005 \mathrm{nM} / \mathrm{mg}$ tissues. In Cerebral cortex of 18 month old mice the MDA levels were $0.23 \pm 0.014 \mathrm{nM}$ of MDA/ $\mathrm{mg}$ tissue. While in hippocampal region the MDA level was $0.14 \pm 0.003 \mathrm{nM} / \mathrm{mg}$ tissues. The MDA levels in cerebral cortex of 24 month old mice were $0.24 \pm 0.015 \mathrm{nM} / \mathrm{mg}$ tissue. In hippocampal region the MDA level was $0.15 \pm 0.01 \mathrm{nM} / \mathrm{mg}$ tissue (Graph 1-3).

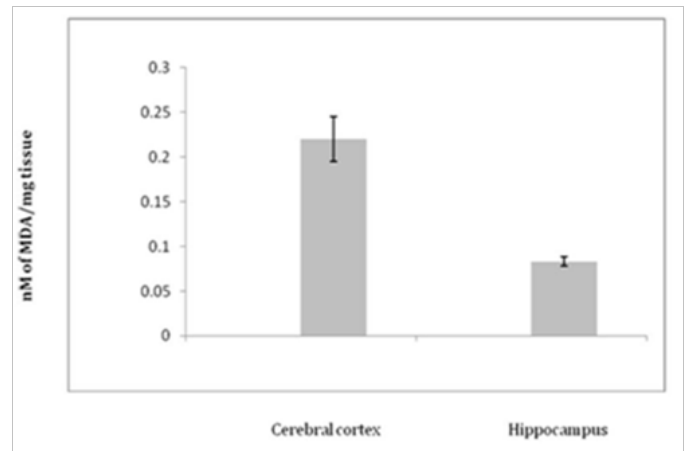

Graph I Lipid peroxidation in Nm of MDA/ mg tissue in cerebral cortex and Hippocampus of 12 month old mice.

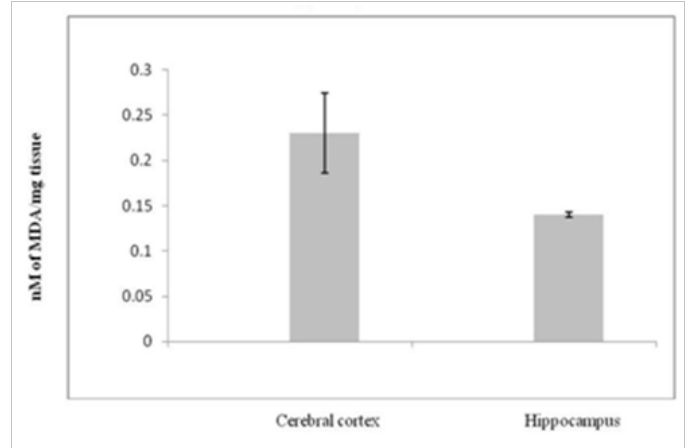

Graph 2 A neuron from the Hippocampus of 24 months old mouse. $\mathrm{EC}$, euchromatin; HC, heterochromatin; $M$, mitochondria; DM, damaged mitochondria; GA, golgi apparatus.

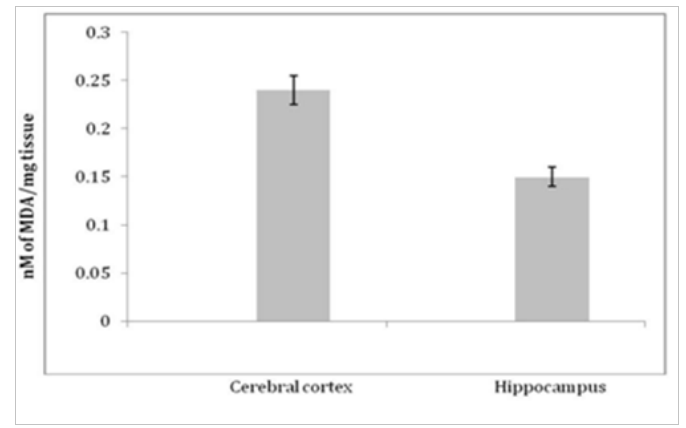

Graph 3 A neuron from the Hippocampus of 24months old mouse. EC, euchromatin; HC, heterochromatin; M, mitochondria; DM, damaged mitochondria; GA, golgi apparatus. 


\section{Results of electron microscopic studies}

A neuron from Cerebral cortex of 12 months old mouse exhibited conspicuous nucleus with prominent euchromatin. The cytoplasm revealed RER, Nissl substance, mitochondria, autophagosome and few lipofuscin granules (Figure 1).

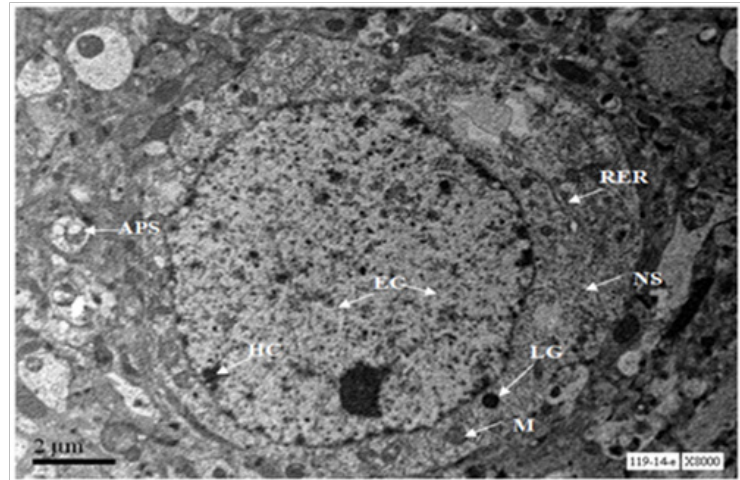

Figure I A neuron from the Cerebral cortex of 12 months old mouse. EC, euchromatin; HC, heterochromatin; APS, autphagosome; LG, lipofuscin granules; M, mitochondrion; NS, nissl substance.

Axons from cerebral cortex at age 12 months showed deposition of electron dense material, well organised neurofilaments (Figure 2).

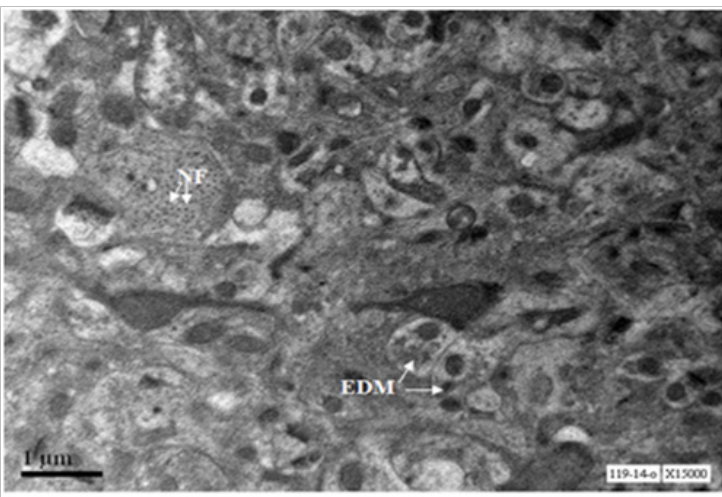

Figure 2 T. S. of axons from Cerebral cortex of 12 months old mouse showing neurofilaments at I5000X. NF, neurofilament; EDM, electron dense material

A neuron from hippocampus of 12 months old mouse exhibited conspicuous nucleus with prominent euchromatin. The cytoplasm revealed RER, Nissl substance, mitochondria, Golgi cisternae and few lipofuscin granules (Figure 3).

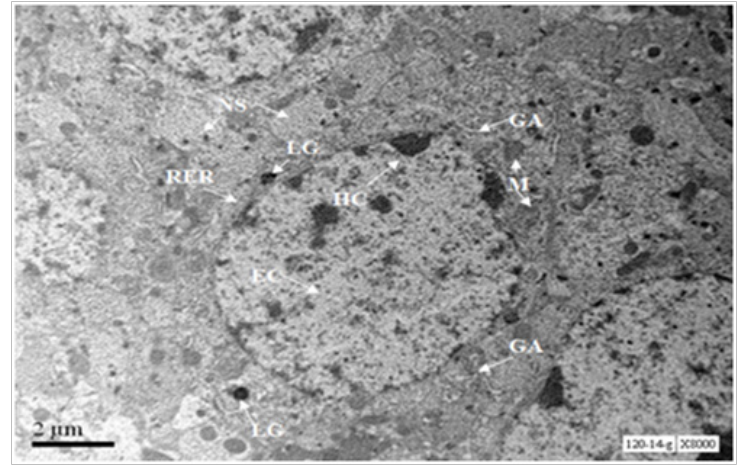

Figure $3 \mathrm{~A}$ neuron from the Hippocampus of 12 months old mouse. EC, euchromatin; HC, heterochromatin; M, mitochondria; NS, nissl substance; LG, lipofuscin granules; RER, rough endoplasmic reticulum; GA, golgi apparatus
Axons from hippocampus at age 12 months show well organised neurofilaments, functional mitochondria and secretary vesicles (Figure 4)

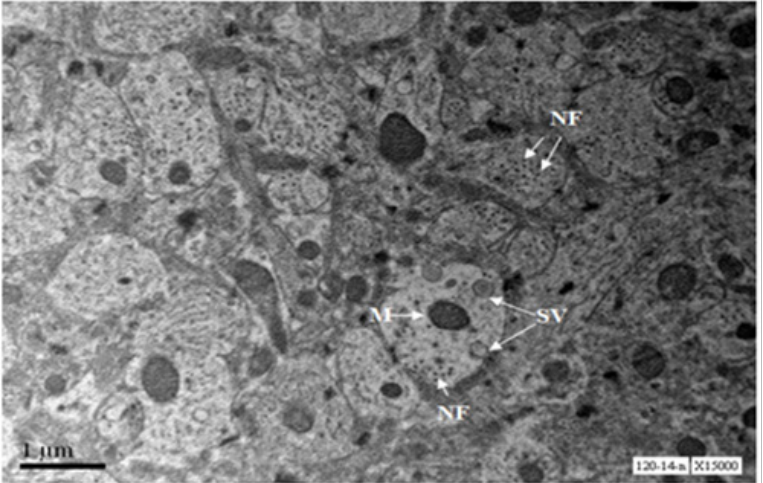

Figure 4 T.S. of axons from Hippocampus of 12 months old mouse showing neurofilament at I5000X. NF, neurofilament; SV, secretory vesicles; M, mitochondria.

A neuron from the cerebral cortex of 18 months old mouse exhibited abundance of heterochromatin. Cytoplasm was loaded with residual bodies. Mitochondria were swollen. Cytoplasm exhibited poor Nissl granules and RER (Figure 5) (Figure 6).

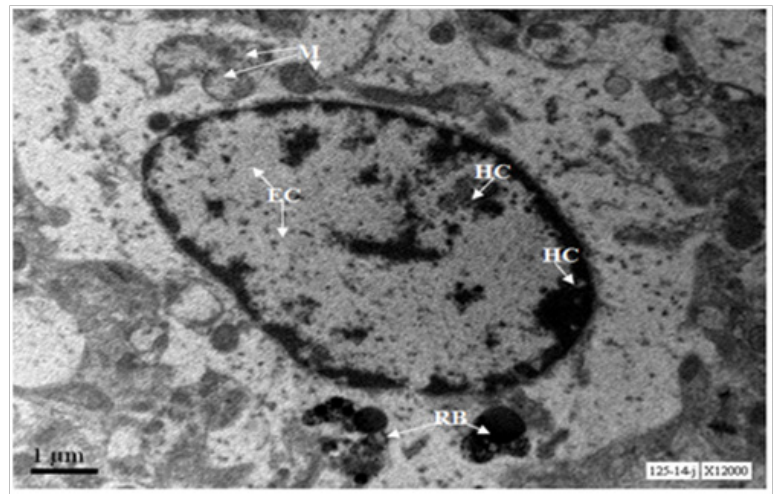

Figure $5 \mathrm{~A}$ neuron from the Cerebral cortex of 18 months old mouse. EC, euchromatin; HC, heterochromatin; $M$, mitochondria; RB, residual bodies

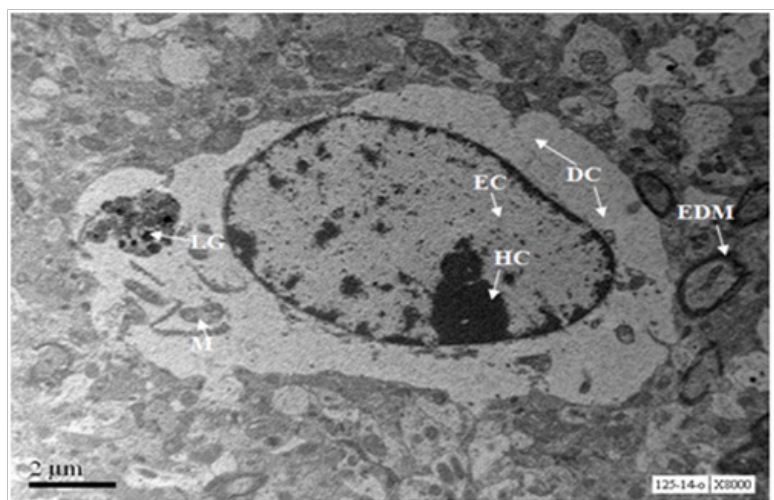

Figure $6 \mathrm{~A}$ neuron from the Cerebral cortex of I8months old mouse. EC, euchromatin; HC, heterochromatin; M, mitochondria; LG, lipofuscin granules; DC, degenerating cytoplasm; EDM, electron dense material.

Axons from cerebral cortex at age 18 months show depostion of external deposits, disorganised neurofilaments and scarce secretary vesicles. One axon shows damaged mitochondrion (Figure 7). 


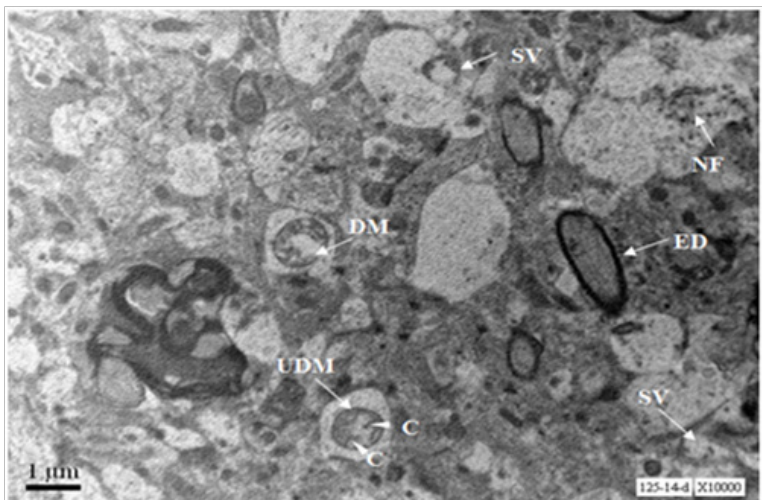

Figure 7 T.S. of axons from Cerebral cortex of 18 months old mouse showing irregularity of neurofilaments and damaged mitochondria at I5000X. NF, neurofilaments, DM, damaged mitochondrion, ED, external deposits, SV, secretory vesicles, UDM, undamaged mitochodrion, C, crista.

A neuron of the hippocampal region of 18 months old mouse exhibited conspicuous nucleus with marginal traces of heterochromatin and abundant euchromatin. Cytoplasm was fully occupied by Niss substance and Rough Endoplasmic Reticulum, however Golgi cisternae were not observed in inflated form. The cytoplasm was loaded with lipofuscin granules. Mitochondria were with normal size and shape without any swelling (Figure 8).

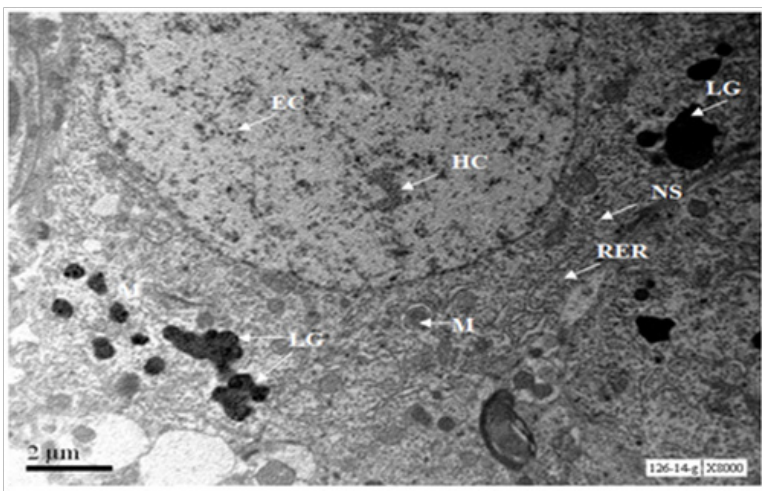

Figure 8 A neuron from Hippocampus of 18 months old mouse. EC, euchromatin, HC, heterochromatin, M, mitochondria, LG, lipofuscin granules, NS, nissl substance, RER, rough endoplamic reticulum.

Axons from hippocampus at age 18 months show no depostion of electron dense material, but little irregularaity in neurofilaments (Figure 9).

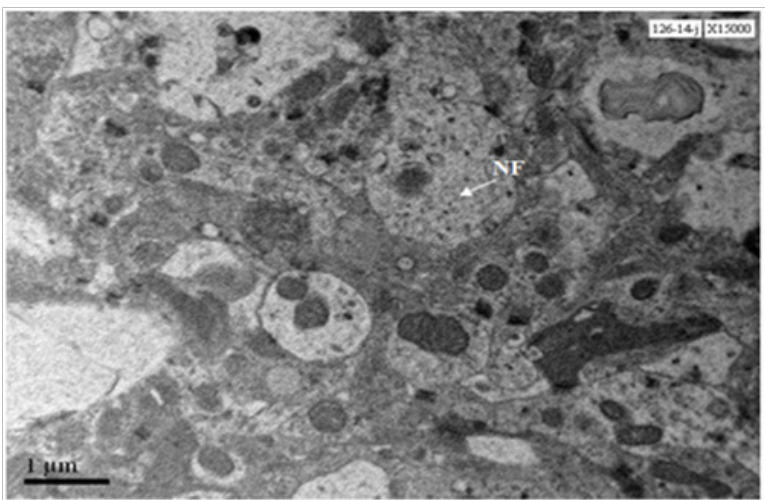

Figure 9 T.S. of axons from Hippocampus of 18 months old mouse at I5000X showing irregularity of neurofilaments. NF, neurofilament
Two neurons from Cerebral cortex of 24 months old mouse exhibit predominance of heterochromatin, nucleus shows early stages of fragmentation; one neurons shows formation of nuclear lobes (neuron in the right side of the panel), whereas the other neuron shows advanced nuclear fragmentation (neuron in the left side of the panel). Both the neurons show loss of cytoplasm, damaged mitochondria, loss of Nissl substance and accumulation of lipofuscin granules (Figure 10). At 20000X magnification lipofuscin granules were conspicuously seen (Figure 11).

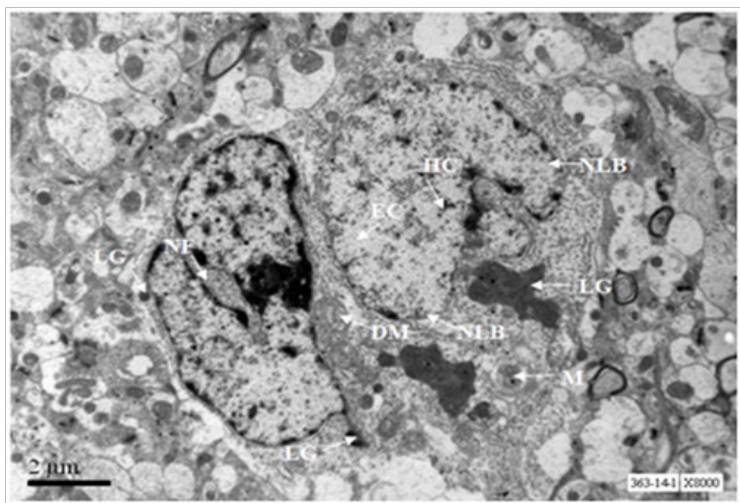

Figure $10 \mathrm{~A}$ neuron from Cerebral cortex of 24 months old mouse showing Nucleus undergoing formation of lobes an initial step of nuclear fragmentation at $8000 X$. EC, euchromatin; HC, heterochromatin; M, mitochondria; LG, lipofuscin granules; NF, nuclear fragmentation; NLB, nuclear lobe.

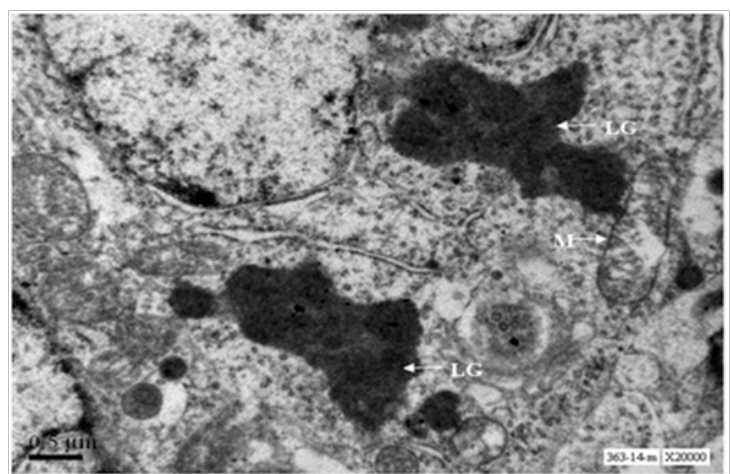

Figure I I A portion of a neuron from the cerebral cortex of 24Months old mouse. LG, lipofuscin granules, M, mitochondrion.

Axons from cerebral cortex at age 24 months show extracellular deposits, axonal rupture from inside and complete loss of regularity of neurofilament architecture (Figure 12).

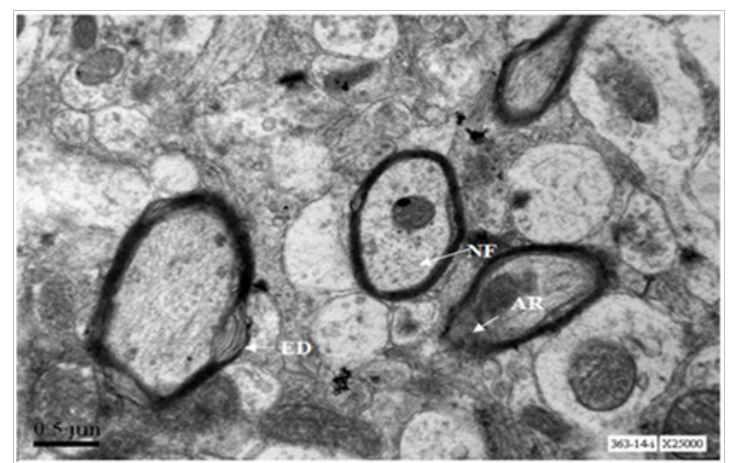

Figure 12 T.S. of axons from Cerebral cortex of 24 months old mouse showing deposits from exterior at 25000X. ED, extracellular deposits; AR, axonal rupture from inside; NF, neurofilament. 
A neuron from hippocampus of 24 months old mouse exhibits prominent nucleus with euchromatin and heterochromatin. Lipofuscin granules were scarce. Cytoplasm exhibits few swollen mitochondria along with normal mitochondria and Golgi cisternae (Figure 13).

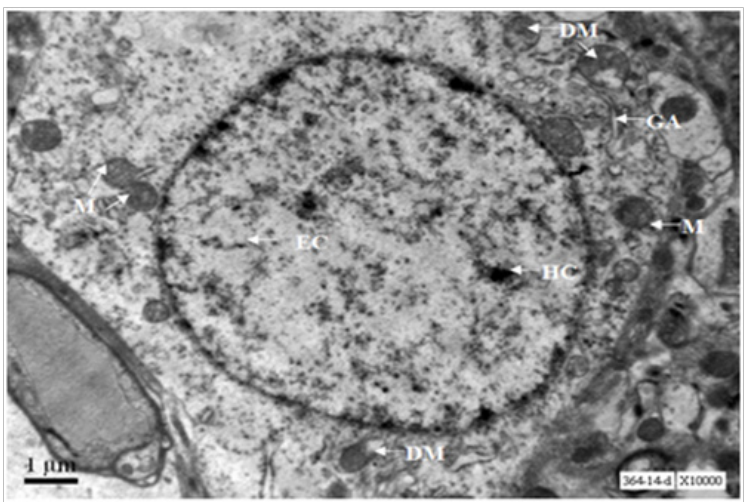

Figure I3 A neuron from the Hippocampus of 24months old mouse. EC, euchromatin; HC, heterochromatin; M, mitochondria; DM, damaged mitochondria; GA, golgi apparatus.

Axons from hippocampus at age 24 months show beginning of deposition of extracellular aggregates (Figure 14) and an axon in the left side of the panel shows rupture.

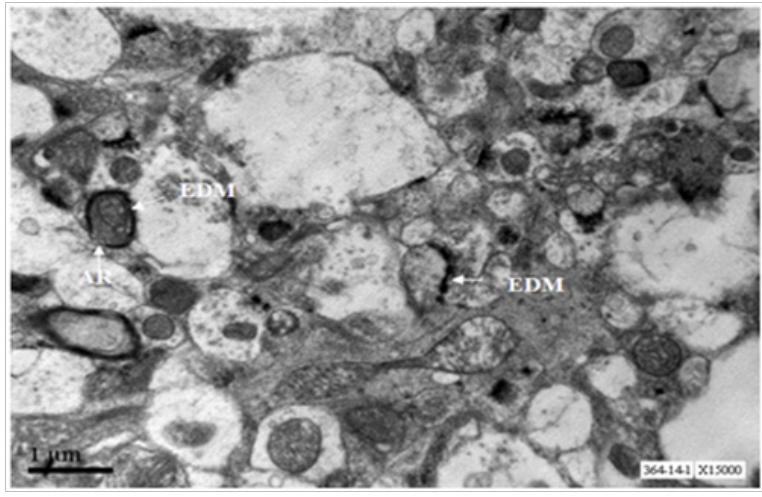

Figure I 4 T.S. of axons from Hippocampus of 24 months old mouse showing deposition of electron dense material. EDM, electron dense material; $A R$, axonal rupture.

The degree of lipid peroxidation was expressed as nM of MDA/ $\mathrm{mg}$ tissue. There was no significant difference in the levels of MDA in the cerebral cortex at the age of 12,18 and 24 months. In the hippocampus, there was highly significant difference $(p<0.001)$ in the levels of MDA at the age of 12 and 18 months as well as 12 and 24 months. The Cerebral cortex exhibited high levels of MDA as compared to hippocampus. There was highly significant difference in the levels of MDA at all ages in the hippocampus when compared to cerebral cortex of respective ages. The cerebral cortex is concerned with perception of stimuli, recognition, planning, short term memory ${ }^{35}$ which get declined during aging. Hippocampus is associated with long term memory which persists even in senescent condition. ${ }^{36}$ In the present investigation, the MDA levels were higher in cerebral cortex than in the hippocampus. This pattern was consistent in 12,18 and 24 months old mice. These results are correlated with decline in neuronal functioning during aging which may be due to difference in the fatty acids composition in the various regions of the brain.

There was no significant increase in the extent of lipid peroxidation in the cerebral cortex from 12 to 18 months and from 18 to 24 months.
This might be due to maximum damage that might have occurred at the age of twelvemonths. This scenario was altogether different in the hippocampus. The maximum oxidative damage that occurred in the hippocampus at the age of 24 months was $37 \%$ lower than in the cerebral cortex at the same age. This indicates that hippocampus is more protected. This could be due to involvement of hippocampus in adult neurogenesis. ${ }^{37,38}$ As new neurons are generated in the hippocampus, they are equipped with more antioxidant defense than the older cerebrocortical neurons.

The Ultrastructural details also reveal that the hippocampus is protected than the cerebral cortex. This is evidenced by loss of cytoplasmic contents such as mitochondria, RER, ribonucleoprotein particles in cerebrocortical neuron. Nucleus shows heavily heterochromatinised region, which suggest transcriptional inactivation. A neuron from hippocampus shows nucleus with predominant euchromatin and scanty heterochromatin suggesting the transcriptional activity of the neuron. Cytoplasm is studded with ribonucleoprotein particles, abundance of RER and mitochondria.

In the transverse section, axons of cerebrocortical neurons of age 18 months show intra-axonal electron dense material which may be beneurofibrillary tangles or aggregates of phosphorylated tau. Recently we have demonstrated tau phosphorylation in the neurons undergoing degeneration in culture. ${ }^{39}$ Axons of hippocampal neurons exhibit well organized neurofilaments. Some axons also show presence of secretary vesicles and undamaged mitochondria. At the age of 24 months, axons of cerebrocortical neurons show heavy extracellular deposition, and few axons show rupture. Whereas, axons of hippocampal neurons show beginning of extracellular deposition and rupture. This suggests that hippocampal neurons are more protected than cerebrocortical neurons at advanced age also. Recently we have also demonstrated that cerebral cortex is more vulnerable to deposition of neurofibrillary tangles than hippocampus. ${ }^{40}$ This may be due to involvement of adult hippocampus in neurogenesis as described by Eriksson et al. ${ }^{41}$ The results of the present study give biochemical and Ultrastructural evidence to the neuronal plasticity in the hippocampus.

\section{Acknowledgements}

Authors are grateful to University Grants Commission, New Delhi for providing financial assistance wide sanction order F.No.4145/2012 (SR) dated 10th July 2012. Authors acknowledge the help of technicians Mr. S.S Bhosale and Mr. Dileep Kanaskar from Department of Electron Microscopy, Jaslok Hospital and Research Centre, Mumbai (India).

\section{Conflict of interest}

The author declares no conflict of interest.

\section{References}

1. Harman D. Aging: a theory based on free radical and radiation chemistry. J Gerontol. 1956;11(3):298-300.

2. Floyd RA. Antioxidants, oxidative stress, and degenerative neurological disorders. Proc Soc Exp Biol Med. 1999;222(3):236-245.

3. Harman D. The aging process: major risk factor for disease and death. Proc Natl Acad Sci U S A. 1991;88(12):5360-5363.

4. Finkel T, Holbrook NJ. Oxidants, oxidative stress and the biology of ageing. Nature. 2000;408(6809):239-247.

5. Harman D. The biologic clock: the mitochondria? J Am Geriatr Soc. 1972;20(4):145-147. 
6. Miquel J, Economos AC, Fleming J, et al. Mitochondrial role in cell aging. Exp Gerontol. 1980;15(6):575-591.

7. Bosco L, Ruvolo G, Morici G, et al. Apoptosis in human unfertilized oocytes after intracytoplasmic sperm injection. Fertil Steril. 2005;84(5):1417-1423.

8. Goldstein S, Meyerstein D, Czapski G. The Fenton reagents. Free Radic Biol Med. 1993;15(4):435-445.

9. Fleming JE, Miquel J, Cottrell SF, et al. Is cell aging caused by respiration-dependent injury to the mitochondrial genome? Gerontol. 1982;28(1):44-53.

10. Hiona A, Leeuwenburgh C. The role of mitochondrial DNA mutations in aging and sarcopenia: implications for the mitochondrial vicious cycle theory of aging. Exp Gerontol. 2008;43(1):24-33.

11. Chomyn A, Attardi G. MtDNA mutations in aging and apoptosis. Biochem Biophys Res Commun. 2003;304(3):519-529.

12. Porter NA, Caldwell SE, Mills KA. Mechanisms of free radical oxidation of unsaturated lipids. Lipids. 1995;30(4):277-290.

13. Bailey DM, Davies B, Young IS, et al. EPR spectroscopic detection of free radical outflow from an isolated muscle bed in exercising humans. $J$ Appl Physiol. 2003;94(5):1714-1718.

14. Dean RT, Fu S, Stocker R, et al. Biochemistry and pathology of radicalmediated protein oxidation. Biochem J. 1997;324(1):1-18.

15. Stadtman ER, Berlett BS. Reactive oxygen-mediated protein oxidation in aging and disease. Chem Res Toxicol. 1997;10(5):485-494.

16. Brunk UT, Terman A. Lipofuscin: mechanisms of agerelated accumulation and influence on cell function. Free Rad Biol Med. 2002;33(5):611-619.

17. Terman A, Gustafsson B, Brunk UT. The lysosomal-mitochondrial axis theory of postmitotic aging and cell death. Chem Biol Interact. 2006;163(1-2):29-37.

18. Krokan HE, Standal R, Slupphaug G. DNA glycosylases in the base excision repair of DNA. Biochem J. 1997;325(1):1-16.

19. Dizdaroglu M, Jaruga P, Birincioglu M, et al. Free radical-induced damage to DNA: mechanisms and measurement. Free Radic Biol Med. 2002;32(11):1102-1115.

20. Kirkinezosa IG, Moraesa CT. Reactive oxygen species and mitochondrial diseases. Semin Cell Dev Biol. 2001;12(6):449-457.

21. Rice-Evans C, Burdon R. Free radical-lipid interactions and their pathological consequences. Prog Lipid Res. 1993;32(1):71-110.

22. Raha S, Robinson BH. Mitochondria, oxygen free radicals, disease and ageing. Trends Biochem Sci. 2000;25(10):502-508.

23. Porter NA, Caldwell SE, Mills KA. Mechanisms of free radical oxidation of unsaturated lipids. Lipids. 1995;30(4):277-290.

24. Montine TJ, Neely MD, Quinn JF, et al. Lipid peroxidation in aging brain and Alzheimers diseases. Free Radic Biol Med. 2002;33(5):620-626.

25. Mooradian AD, Lung CC, Shah G, et al. Age related changes in tissue content of malondialdehyde-modified proteins. Life Sci. 1994;55(20):1561-1566.
26. Lovell MA, Ehmann WD, Butler SM, et al. Elevated thiobarbituric acid-reactive substances and antioxidant enzyme activity in the brain in Alzheimer's disease. Neurology. 1995;45(8):1594-1601.

27. Mark RJ, Hensley K, Butterfield DA, et al. Amyloid beta-peptide impairs ion-motive ATPase activities: evidence for a role in loss of neuronal Ca2+ homeostasis and cell death. J Neurosci. 1995;15(9):6239-6249.

28. Mark RJ, Lovell MA, Markesbery WR, et al. A role for 4 hydroxynonenal, an aldehydic product of lipid peroxi-dation, in disruption of ion homeostasis and neuronal death induced by amyloid b-peptide. $J$ Neurochem. 1997;68(1):255-264.

29. Jellinger KA. The role of iron in neurodegeneration: prospects for pharmacotherapy of Parkinson's disease. Drugs Aging. 1999;14(2):115140.

30. Pratico D, Uryu K, Leight S, et al. Increased lipid peroxidation precedes amyloid plaque formation in an animal model of Alzheimer amyloidosis. $J$ Neurosci. 2001;21(12):4183-4187.

31. Butterfield DA, Bader Lange ML, Sultana R. Involvements of the lipid peroxidation product, HNE, in the pathogenesis and progression of Alzheimer's disease. Biochim Biophys Acta. 2010;1801(8):924-929.

32. Basu AK, Marnett LJ. Unequivocal demonstration that malondialdehyde is a mutagen. Carcinogenesis. 1983;4(3):331-333.

33. Michiels C, Remacle J. Cytotoxicity of linoleic acid peroxide, malondialdehyde and 4-hydroxynonenal towards human fibroblasts. Toxicol. 1991;66(2):225-234.

34. Wills ED. Mechanisms of lipid peroxide formation in animal tissues. Biochem J. 1966;99(3):667-676.

35. Kandel ER, Schwartz JH, Jessell TM. Principles of Neural Science. 4th ed. USA: McGraw-Hill; 2000. 324 p.

36. Small SA, Chawla MK, Buonocore $\mathrm{M}$, et al. Imaging correlates of brain function in monkeys and rats isolates a hippocampal subregion differentially vulnerable to aging. Proc Natl Acad Sci USA. 2004;101(18):7181-7186.

37. Kempermann G, Kuhn HG, Gage FH. More hippocampal neurons in adult mice living in an enriched environment. Nature. 1997;386(6624):493495.

38. Stangl D, Thuret S. Impact of diet on adult hippocampal neurogenesis. Genes Nutr. 2009;4(4):271-282.

39. Bele MS, Gajare KA, Deshmukh AA. Caloric restriction mimetic 2 deoxyglucose maintains cytoarchitecture and reduces tau phosphorylation in primary culture of mouse hippocampal pyramidal neurons. In Vitro Cell Dev Biol Anim. 2015;51(6):546-55.

40. Pathan KH, Gajare KA, Deshmukh AA. Cytopathological study of neurofibrillary tangles in cerebral cortex and hippocampus of swiss albino mouse:Mus Musculus. J Pharma Biol. 2015;5(4):314-319.

41. Eriksson PS, Perfilieva E, Bjork-Eriksson T, et al. Neurogenesis in the adult human hippocampus. Nat Med. 1998;4(11):1313-1317. 